\title{
Cross-Layer Solutions for Cooperative Medium Access Control Protocols
}

\author{
Alessandro Crismani, Fulvio Babich \\ Dipartimento di Elettrotecnica, Elettronica ed Informatica \\ Università di Trieste, Via A. Valerio 10, 34127 Trieste, Italy \\ Tel: +39(040)558-7146; Fax: +39(040)558-3460 \\ e-mail: alessandro.crismani@phd.units.it, babich@units.it
}

\author{
Lajos Hanzo \\ School of Electronics and Computer Science \\ University of Southampton, SO17 1BJ, UK \\ e-mail: 1h@ecs.soton.ac.uk
}

\begin{abstract}
Recent studies have shown that designing a Medium Access Control (MAC) protocol combined with a cooperative approach may improve the attainable network throughput, despite reducing the mean packet delay. In this paper we design a MAC scheme adopting cooperative physical layer aided cross-layer techniques. We consider a popular cooperative MAC protocol, namely the CoopMAC technique of Liu $e t$ al., which is improved by facilitating cooperative signal combining at the destination and employing two relays in the context of a successive relaying technique. The performance of the proposed scheme is evaluated by Monte-Carlo simulations. We demonstrate that a cross-layer design further improves the performance gain provided by the CoopMAC protocol over that of the legacy 802.11 Distributed Coordination Function (DCF), enhancing both the achievable network throughput and the outage probability.
\end{abstract}

\section{INTRODUCTION}

In a cooperative scenario nodes equipped with a single antenna share their information to form a virtual antenna array (VAA) for achieving the maximum attainable spatial diversity provided by independently faded antenna elements.

Whilst the literature of physical layer cooperative techniques is rich [1], only a few papers considered the design of cooperative Medium Access Control (MAC) protocols [2], [3], [4], [5]. In a cooperative scenario data are transmitted from the source to a relay, which then forwards them to the destination. The Cooperative Linear Dispersion Codes (CLDCs) of [1] were designed with the aim of eliminating the effects of antenna-element correlation typically imposed on co-located antenna elements of space-time codes, where the VAA elements were constituted by the mobiles' single antennas. However, in the amplify-and-forward schemes of [1] no attempt was made to simultaneously exploit the source's transmissions at the destination. In a more sophisticated scenario the destination listens to both the source and relay transmissions, which may be combined and jointly decoded. Furthermore, references [2], [3], [4], [5] rely on the simplifying assumption of having a perfect physical layer. A CrossLayer (CL) approach designed for the CoopMAC protocol of [6] was presented in [3], although the effect of channel coding was not investigated and the adaptive transmission rate selection algorithm did not exploit the additional information provided by the joint decoding of the signals gleaned from the source $(\mathrm{S})$ and the relay $(\mathrm{R})$ at the destination (D).

\section{A. Novel contribution}

In this paper we intrinsically amalgamate physical-layer cooperative techniques with the CoopMAC [6]. We contrive a joint detection scheme for the direct and relayed signals combined with

The financial support of the EK under the auspices of the Pan-European Optimix project, that of the EPSRC UK and of the University of Trieste is gratefully acknowledged developing a novel modem mode/transmission rate selection regime. The proposed adaptive scheme allows the relay to select the most appropriate transmission rate, which guarantees maintaining a fixed Block Error Ratio (BLER) at the destination, despite facing of time-variant interference and propagation conditions. We mitigate the multiplexing loss imposed by a single-relay-aided cooperative scenario due to the half duplex constraint of 802.11 stations, which cannot simultaneously transmit and receive. More explicitly, we employ two relays in our successive relaying aided scheme [7]. To incorporate successive relaying, we propose a modified CoopMAC scheme, which facilitates communications between the source and two specifically selected relays in order to set up a twin-relayscenario. The performance of the proposed schemes is evaluated using bit-by-bit Monte-Carlo simulations.

We will demonstrate that by introducing the proposed rate selection regime and employing successive relaying in the CoopMAC context, we can further increase the achievable network throughput, whilst simultaneously reducing the outage probability.

The rest of this paper is organized as follows. The system model used in our simulations is described in Section II. The rate selection algorithm and the successive relaying protocol are detailed in Section III. In Section IV a rudimentary theoretical analysis is performed for calculating the outage probability of the different schemes. Our simulation results and discussions will be provided in Section V. Finally, we conclude in Section VI.

\section{THE SYSTEM UNDER EVALUATION}

We model an infrastructure based 802.11 wireless network, where every station transmits its data to the Access Point (AP). The communicating stations were located on a circle with a normalized radius of $R=1$, while the AP was placed in the middle of the circle. The number of stations $(N)$ was varied between $N=5$ and 35 , where $N$ incorporated both the number of transmitting nodes and the AP. For example, $N=7$ implies having six terminals plus the AP.

\section{A. Signal-to-noise ratio between stations and propagation model}

The communication channel was characterized either by Additive White Gaussian Noise (AWGN) or by non-dispersive Rayleigh fading. Slow Rayleigh fading was assumed, hence the fading channel gain $\gamma$ was constant for a frame's transmission. More explicitly, the SNR of every frame's transmission between nodes "a" "and b" was determined by the instantaneous Rayleigh fading envelope, which may be formulated as as:

$$
\mathrm{SNR}_{a, b}=\gamma_{a, b} \overline{\mathrm{SNR}_{a, b}},
$$




\begin{tabular}{|l|c|}
\hline Bit rate & Modulation \\
\hline $1 \mathrm{Mbps}$ & BPSK \\
$2 \mathrm{Mbps}$ & QPSK \\
$3 \mathrm{Mbps}$ & 8-PSK \\
$4 \mathrm{Mbps}$ & 16-QAM \\
$5 \mathrm{Mbps}$ & $4-12-16 \mathrm{APSK}$ \\
$6 \mathrm{Mbps}$ & 64-QAM \\
\hline
\end{tabular}

TABLE I

MODULATIONS USED TO OBTAIN DIFFERENT BIT RATES.

\begin{tabular}{|c|c|c|c|c|c|}
\hline \multicolumn{5}{|c|}{ Instantaneous SNR } & Chosen modulation \\
\hline $\mathrm{SNR}_{\mathrm{dB}}$ & $<$ & $1.60 \mathrm{~dB}$ & & & Outage \\
\hline $1.60 \mathrm{~dB}$ & $\leq$ & $\mathrm{SNR}_{\mathrm{dB}}$ & $<$ & $4.60 \mathrm{~dB}$ & BPSK \\
\hline $4.60 \mathrm{~dB}$ & $\bar{s}$ & $\mathrm{SNR}_{\mathrm{dB}}$ & $<$ & $8.77 \mathrm{~dB}$ & QPSK \\
\hline $8.77 \mathrm{~dB}$ & $\leq$ & $\mathrm{SNR}_{\mathrm{dB}}$ & $<$ & $10.82 \mathrm{~dB}$ & 8PSK \\
\hline $10.82 \mathrm{~dB}$ & $\leq$ & $\mathrm{SNR}_{\mathrm{dB}}$ & $<$ & $13.84 \mathrm{~dB}$ & 16QAM \\
\hline $13.84 \mathrm{~dB}$ & $\bar{\Sigma}$ & $\mathrm{SNR}_{\mathrm{dB}}$ & $<$ & $16.20 \mathrm{~dB}$ & 4-12-16APSK \\
\hline $\mathrm{SNR}_{\mathrm{dB}}$ & $\geq$ & $16.20 \mathrm{~dB}$ & & & 64QAM \\
\hline
\end{tabular}

TABLE II

MODULATION MODES VERSUS THE INSTANTANEOUS SNR.

where $\gamma_{a, b}$ models the Rayleigh fading attenuation and $\overline{\mathrm{SNR}_{a, b}}$ is the mean SNR characterizing the AWGN. We fixed the mean SNR value $\overline{\mathrm{SNR}}$ at a normalized distance of 1 meter and hence the mean SNR between nodes "a" and "b" was calculated as:

$$
\overline{\mathrm{SNR}_{a, b}}=\frac{\overline{\mathrm{SNR}}}{d(a, b)^{\alpha}}
$$

where $d(a, b)$ is the Euclidean distance between nodes "a" and "b", while $\alpha$ is the path-loss exponent, which was set to $\alpha=3$.

\section{B. Data rate selection for direct and relay-aided links}

In this study the transmission rates are chosen from a discrete set, which is formed by six modulation modes associated with a fixed symbol duration, as shown in Table I.

Channel coding is also considered and data are encoded by a Parallel Concatenated Single Parity Check Product Code (PC-SPCPC) [8], [9], [10], which are capable of performing within $1 \mathrm{~dB}$ from the sphere-packing limit [1], [9]. The switching thresholds employed to select the modulation modes correspond to the average Signal to Noise Ratio (SNR) required for achieving a BLER of 0.1 at the destination. For the PC-SPC-PC code a block length of 9000 bits (arranged in a $500 \times 18$ matrix) and a coding rate of $R_{C}=0.75$ combined with the thresholds of Table II was used. These thresholds were determined by simulations using the PC-SPCPC codes advocated, but they may also be theoretically validated using the random coding exponent approach presented in [11] for BPSK modulation and in [9] for more generic modulation schemes.

In order to mitigate error propagation, a higher SNR may be required on the source-relay (SR) link, since a lower BLER an BER are tolerated at the relay, especially in decode-and-forward scenarios. A value of $\xi=1.5$ is used for quantifying the relay-aided SNR improvement, which scales every threshold in the system, before comparing the instantaneous SNR to them.

For example, an instantaneous SNR of $\mathrm{SNR}_{a, b}=5 \mathrm{~dB}$ will result in the choice of QPSK on both the source-destination (SD) and the relay-destination (RD) links, while BPSK would be employed on the (SR) link.

\section{The choice of cooperating partners and their activation}

Let $R_{s, r_{j}}$ be the rate available between the source station and the $i^{\text {th }}$ relay, while $R_{r_{j}, d}$ be the rate available between the $j^{\text {th }}$ relay and the destination. The cooperating partner selection criterion is the following. Choose relay $i$, where we have:

$$
i=\underset{j \in \text { helpers }}{\operatorname{argmin}}\left(\frac{1}{R_{s, r_{j}}}+\frac{1}{R_{r_{j}, d}}\right),
$$

where the set helpers represents the available relays (in this paper every node connected to the network may forward packets from a source to a destination). We assume that the source knows the SNRs required for performing the rate selection, which can be estimated during unallocated time-slots, as detailed in [6] for the CoopTable ${ }^{1}$.

The decision on whether to cooperate or not is based on an overall transmission duration minimization criterion formulated as follows, which may also be viewed as a throughput maximization problem. Let $T^{\text {dir }}$ be the time required to transmit a net payload of a data file from the source to the destination on the SD link, while $T_{j}^{\text {coop }}$ represents the transmission duration using the $j^{\text {th }}$ relay in a relayaided scenario. If we have

$$
\min _{j \in \text { helpers }}\left(T_{j}^{\text {coop }}\right)<T^{\mathrm{dir}}
$$

the cooperation is activated, otherwise classic direct transmission takes place.

\section{CROSS-LAYER TECHNIQUeS FOR THE COOPMAC PROTOCOL}

In the legacy CoopMAC protocol of [6] the destination uses only the signal received from the relay. However, due to the broadcast nature of radio communications, the destination listens also to the source station's transmission. Although its received signal may be low (otherwise there would be no need to cooperate), it may still improve the reliability of the decoded data forwarded by the relay.

The proposed scheme, which we refer to as signal combining aided CoopMAC, facilitates this joint decoding by adding the LogLikelihood Ratios (LLRs) of bits received from both the source and the relay.

Since the destination processes also the signal received from the source, the SNR of the RD link required to achieve a BLER of 0.1 is reduced. We propose a simple modem mode selection rule based on a linear interpolation, in order to choose the RD link's rate, given $\mathrm{SNR}_{r, d}$ and $\mathrm{SNR}_{s, d}$.

Let the data between the source and the relay be transmitted using modulation mode $i$ (which is the highest-throughput mode available on that link) and let $T_{i}$ be the SNR threshold, above which the $i^{t h}$ modulation mode is activated. The modulation mode $j$ can be used on the RD link, if we have:

$$
\mathrm{SNR}_{r, d}>T_{j}^{\mathrm{lin}}=T_{j}\left(1-\frac{\mathrm{SNR}_{s, d}}{T_{i}}\right)
$$

where $T_{j}^{\text {lin }}$ represents the new threshold for modulation mode $j$ derived by linear interpolation.

To justify this modem mode selection rule Figure 1 compares the SNR required on the RD link, obtained by simulations for the PCSPC-PC code employed to the proposed linear interpolation based threshold, given a fixed an SNR on the SD link. The quantities used in 5 and in Figure 1 are real valued and not logarithmic ones. Let, for example, the instantaneous SNRs be $\mathrm{SNR}_{s, d}=4 \mathrm{~dB}, \mathrm{SNR}_{s, r}=$ $10.67 \mathrm{~dB}$ and $\mathrm{SNR}_{r, d}=15.51 \mathrm{~dB}$. Without combining the 8PSK mode will be chosen on the SR link, while the 4-12-16APSK mode

${ }^{1}$ CoopMAC stores both the identity of the eligible relays along with their MAC addresses, as well as the SR and RD rates in a table referred to as CoopTable, which is populated by passively listening to the RTS-CTS frames exchanges 
will be chosen on the RD link. With combining the 64QAM mode is chosen on the RD link as:

$$
\begin{aligned}
T_{64 \mathrm{QAM}}^{\mathrm{lin}} & =T_{64 \mathrm{QAM}}\left(1-\frac{\mathrm{SNR}_{s, d}}{T_{8 P S K}}\right)=41.68\left(1-\frac{2.51}{7.54}\right)= \\
& =27.8=14.44 \mathrm{~dB}
\end{aligned}
$$

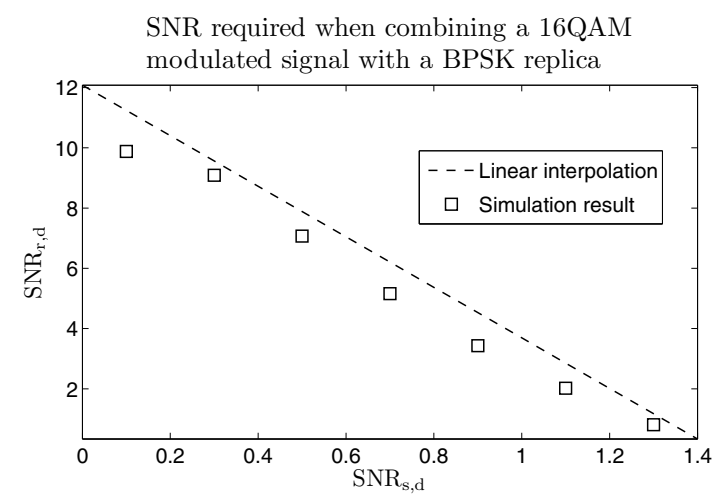

Fig. 1. The SNR required for a $16 \mathrm{QAM}$ modulated signal on the RD link when combined with a BPSK signal on the SD link.

It has to be noted that choosing the highest rate on the SR link may not be the optimal solution. More particularly, using BPSK modulation is expected to provide the best outage probability, as we will detail in Section IV. Conversely, in order to minimize the overall transmission duration, the transmission rate on the SR link should be chosen, so that the $\left(R_{s, r}, R_{r, d}\right)$ combination provides the minimum data transfer duration. Nonetheless, in this study, always the highest achievable rate is activated at a given SNR.

\section{A. Successive relaying aided CoopMAC}

In the three-terminal cooperative scenario considered, a significant multiplexing loss will be incurred compared to direct transmissions due to the half duplex constraint of 802.11 stations.

To mitigate this time loss, a successive relaying protocol was proposed in [7]. Kong et al. [12] employed a successive relaying technique, which was capable of approaching the relay-aided link's capacity. In order to support successive relaying, two relays are needed and the corresponding packet transmission ensues as follows.

Let us divide the packet to be transmitted in $M$ segments and let $P_{i}$ be the $i^{t h}$ one. Transmissions take places in $(M+1)$ consecutive phases:

- in the first phase $S$ transmits $P_{1}$; the $R_{1}$ and $D$ listen to the source's transmission, while $R_{2}$ remains idle;

- in the second phase $S$ transmits $P_{2}$ and $R_{1}$ forwards $P_{1} ; R_{2}$ receives $P_{2}$ from $S$ and $D$ receives $P_{2}$ from $S$ as well as $P_{1}$ from $R_{1}$;

- in the third phase $S$ transmits $P_{3}$ and $R_{2}$ forwards $P_{2} ; R_{1}$ receives $P_{3}$ from $S$ and $D$ receives $P_{3}$ from $S$ as well as $P_{2}$ from $R_{2}$;

- this process continues in this way up to phase $M$;

- in phase $(M+1)$ both $S$ and $R_{1}$ (or $R_{2}$ ) remain silent, while $R_{2}$ (or $R_{1}$ ) forwards $P_{M}$.

Using this successive relaying technique the multiplexing loss is reduced to the value of $\frac{M+1}{M}$, which approaches unity, if $M$ is sufficiently high.

\begin{tabular}{|l|r|r|}
\hline \multicolumn{2}{|l|}{ Instantaneous SNR } & Chosen modulation \\
\hline $\mathrm{SNR}_{\mathrm{dB}}<2.18 \mathrm{~dB}$ & & Outage \\
$2.18 \mathrm{~dB} \leq \mathrm{SNR}_{\mathrm{dB}}<5.18 \mathrm{~dB}$ & BPSK \\
$5.18 \mathrm{~dB} \leq \mathrm{SNR}_{\mathrm{dB}}<9.47 \mathrm{~dB}$ & QPSK \\
$9.47 \mathrm{~dB} \leq \mathrm{SNR}_{\mathrm{dB}}<11.45 \mathrm{~dB}$ & 8PSK \\
$11.45 \mathrm{~dB} \leq \mathrm{SNR}_{\mathrm{dB}}<14.49 \mathrm{~dB}$ & 16QAM \\
$14.49 \mathrm{~dB} \leq \mathrm{SNR}_{\mathrm{dB}}<16.93 \mathrm{~dB}$ & 4-12-16APSK \\
$\mathrm{SNR}_{\mathrm{dB}} \geq 16.93 \mathrm{~dB}$ & & 64QAM \\
\hline
\end{tabular}

TABLE III

MODULATION MODES VERSUS THE INSTANTANEOUS SNR FOR SUCCESSIVE RELAYING AIDED COMMUNICATIONS.

In order to use the above-mentioned successive relaying scheme the source has to address in its Ready to Send (RTS) message the two highest-SNR relay stored in its CoopTable. The two best relays, namely $i$ and $j$, are chosen according to Equation 3. Similarly to the legacy CoopMAC [6], we introduced a Helper ready to Send (HTS) message, which is issued by both of the relays in order to confirm that they are willing to cooperate. Then the ensuing frame exchange of the successive transmission regime is shown in Figure 2.

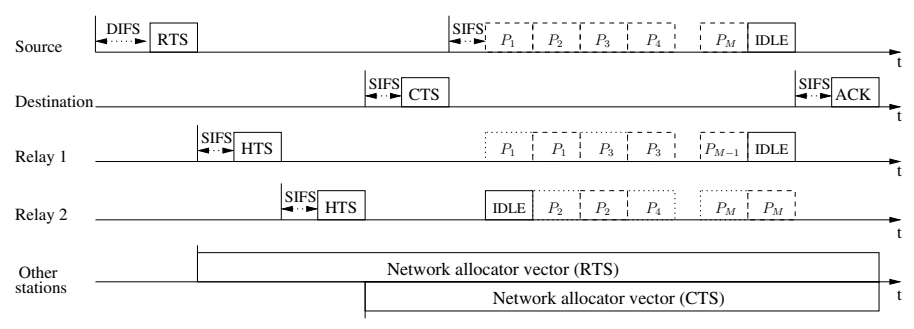

Fig. 2. Frames exchange in successive relaying aided cooperation; dashed boxes represent transmitted packet segments, while dotted boxes represent received ones.

In order to decode the signal arriving from both the relay and the source during the same phase, the destination has to perform Successive Interference Cancellation (SIC) . A low-complexity SIC algorithm was used in [12], [13], while in this study perfect interference cancellation is assumed. In order to mitigate the interference between the relays, it is assumed that they also employ a SIC algorithm to recover the source frames.

When using the successive relaying protocol of Figure 2, the channel encoded block length may vary, since each packet segment is encoded separately. The corresponding modulation mode thresholds of the rate selection algorithm using $M=10$ are shown in Table III. The successive relaying scheme of Figure 2 may be used both with and without signal combining at the destination. When signal combining is not enabled, the destination uses only the segments forwarded by the relays. Conversely, when activating signal combining, the destination adds the LLRs of the bits received from both the source and the relays. Furthermore, when employing signal combining, the rates of the RD link for both relays are chosen according to the linear interpolation procedure introduced in Equation (5).

To decide whether to invoke cooperation or not, the source station evaluates the expected transmission duration of a direct transmission for the best single relay, as well as for the best relay pair using successive transmissions. The specific scheme, which provides the fastest transmission at the highest average rate is activated. 


\section{TheORETICAL OUTAGE ANALYSIS}

In this section we evaluate the outage probability for the different proposed schemes.

A direct transmission is deemed to be in outage, when we have:

$$
\mathrm{SNR}_{s, d}=\gamma_{s, d} \overline{\mathrm{SNR}_{s, d}}<T_{\mathrm{BPSK}} .
$$

Based on Equation (6) one can derive the outage probability as:

$$
P_{\mathrm{out}}^{\mathrm{DCF}}=1-\exp \left(-\frac{T_{\mathrm{BPSK}}}{\overline{\mathrm{SNR} s, d}}\right) \text {. }
$$

Enabling cooperation in a 802.11 network provides a way to reduce the outage probability. More explicitly, when the direct link is in outage (i.e. we have $\mathrm{SNR}_{s, d}<T_{\mathrm{BPSK}}$ ), a packet may be successfully delivered by relay aided transmission, provided that at least one high-quality relay is available. Furthermore, the probability of outage decreases when more stations are connected to the network, as it is more probable to find a relay capable of successfuly forwarding a packet to the destination when no direct transmission is available.

The outage probability of the SR link for the $i^{t h}$ relay can be formulated as:

$$
P_{\text {out }}^{s, r_{i}}=1-\exp \left(\xi \frac{T_{\mathrm{BPSK}}}{\overline{\mathrm{SNR}_{s, r_{i}}}}\right)
$$

and the outage probability of the RD link is given by:

$$
P_{\text {out }}^{r_{i}, d}=1-\exp \left(\frac{T_{\mathrm{BPSK}}}{\overline{\mathrm{SNR}_{r_{i}, d}}}\right) .
$$

In the legacy CoopMAC [6] these two links are independent, hence the outage probability for the $i^{\text {th }}$ relay is given by:

$$
P_{\text {out }}^{\mathrm{RELAY}_{i}}=1-\left(1-P_{\text {out }}{ }^{s, r_{i}}\right)\left(1-P_{\text {out }}{ }^{r_{i}, d}\right) .
$$

Finally the overall communication session is in outage, when both the direct link and all the eligible relay-aided links are in outage. This probability can be calculated as

$$
P_{\text {out }}^{\text {Legacy CoopMAC }}=P_{\text {out }}^{\mathrm{DIR}} \times \prod_{i \in \text { helpers }} P_{\text {out }^{\mathrm{RELAY}_{i}} .}^{\text {R }^{2}}
$$

In the signal combining aided CoopMAC the new RD rate selection depends both on the modulation mode used in the SR link as well as on the value of $\mathrm{SNR}_{s, d}$. Hence, the outage probability of a relayaided link may be conditioned on the value of $\gamma_{s, d}$ and on the modulation mode $j$ chosen on the SR link, yielding:

$$
\begin{aligned}
& P\left\{\text { out }^{\mathrm{RELAY}_{i}} / m_{j}, \gamma_{s, d}\right\}= \\
& =P\left\{\gamma_{r, d} \overline{\mathrm{SNR}_{r, d}}<T_{\mathrm{BPSK}}\left(1-\frac{\gamma_{s, d}}{T_{j}}\right)\right\} \\
& =1-\exp \left[\frac{T_{\mathrm{BPSK}}}{\overline{\mathrm{SNR}_{r, d}}}\left(1-\gamma_{s, d} \frac{\overline{\mathrm{SNR}}}{T_{j}}\right)\right] .
\end{aligned}
$$

As briefly mentioned in Section III, it can be observed that the minimum value of $P\left\{\right.$ out $\left.^{\mathrm{RELAY}_{i}} / m_{j}, \gamma_{s, d}\right\}$ is achieved, when $T_{j}$ BPSK modulation is used on the SR link.

The probability of outage for a generic relay conditioned on the value of $\gamma_{s, d}$ is calculated by invoking the following simple probability relationship:

$$
\begin{aligned}
P\left\{\text { out }^{\mathrm{RELAY}_{i}} / \gamma_{s, d}\right\}= & \sum_{j=0}^{K} P\left\{\text { out }^{\mathrm{RELAY}_{i}} / m_{j}, \gamma_{s, d}\right\} \times \\
& \times P\left\{m_{j}\right\},
\end{aligned}
$$

where $K$ is the number of different modulation modes in the set and $P\left\{m_{j}\right\}$ is the probability of choosing the $j^{t h}$ modulation mode on

\begin{tabular}{|l|r|}
\hline Parameter & Value \\
\hline SIFS & $10 \mu \mathrm{s}$ \\
DIFS & $50 \mu \mathrm{s}$ \\
Slot time & $20 \mu \mathrm{s}$ \\
Maximum number of retransmissions & 6 \\
MAC frame length & $9000 \mathrm{bits}$ \\
ACK Timeout & $300 \mu \mathrm{s}$ \\
CTS Timeout & $300 \mu \mathrm{s}$ \\
CWmin & 16 \\
CWmax & 512 \\
\hline
\end{tabular}

TABLE IV

802.11 PARAMETERS USED IN SIMULATIONS.

the SR link ( $m_{0}$ means that the link is in outage), which may be calculated as:

$$
\begin{aligned}
P\left\{m_{j}\right\} & =P\left\{\xi T_{j} \leq \gamma_{s, r} \overline{\mathrm{SNR}_{s, r}}<\xi T_{j+1}\right\}= \\
& =\exp \left(-\frac{\xi T_{j}}{\overline{\mathrm{SNR}_{s, r}}}\right)-\exp \left(-\frac{\xi T_{j+1}}{\mathrm{SNR}_{s, r}}\right),
\end{aligned}
$$

where $T_{0}=0, T_{K+1}=+\infty$ and $P\left\{\right.$ out $\left.^{\text {RELAY }_{i}} / m_{0}, \gamma_{s, d}\right\}=1$. Finally, the value of $\gamma_{s, d}$ satisfies $\gamma_{s, d}<\frac{T_{\mathrm{BPSK}}}{\mathrm{SNR}_{s, d}}=\delta$, when the direct link is in outage. Hence, the overall outage probability is given by:

$$
\begin{aligned}
P_{\text {out }}^{\text {Combining CoopMAC }}= & \int_{0}^{\delta} \prod_{\substack{i \in \text { helpers }\\
}} P\left\{\operatorname{out}^{\mathrm{RELAY}_{i}} / \gamma_{s, d}\right\} \times \\
& \times f_{\gamma_{s, d}}\left(\gamma_{s, d}\right) d \gamma_{s, d} .
\end{aligned}
$$

The outage probabilities of the successive relaying protocols are the same as those of their single-relay counterparts. This fact follows from observing that in order to use a successive relaying regime two sufficiently high-quality relays are needed, so that at least one beneficial relay link exists.

\section{Performance of THE PRoposed SCHEMES}

Simulations have been performed for evaluating the mean throughput and the outage probability of the schemes studied. Different mean SNRs $(\overline{\mathrm{SNR}})$ have been considered at the distance of $1 \mathrm{~m}$. Table IV provides a summary of the 802.11 parameters used in our simulations.

A discrete-time simulator written in $\mathrm{C}++$ and employing IT++ libraries was used. The core of the simulator is the transmission routine, which performs bit-by-bit transmissions and embeds the proposed physical layer techniques in the 802.11 network. The corresponding simulation results are shown in Figures 3, 4 and 5. Observe from the figures that enabling cooperation in a wireless network is capable of increasing the achievable throughput, while simultaneously decreasing the outage probability. Figure 4 shows that successive relaying has the potential of reducing the multiplexing loss incurred in a single-relay-aided scenario and of providing a throughput of about $9.6 \%$ higher than that of the single-relay assisted scenario and about $23.8 \%$ higher than the DCF protocol for a network of $N=20802.11$ stations.

When adopting the proposed rate selection regime for the RD link, the attainable throughput may be further improved, providing a gain of about $11.7 \%$ with respect to the single-relay scenario and $25.6 \%$ with respect to the DCF protocol for $N=20$ stations and $\overline{\mathrm{SNR}}_{\mathrm{dB}}=10 \mathrm{~dB}$. Furthermore, the rate selection algorithm advocated assists in decreasing the outage probability, which was reduced to 0.24 in comparison to 0.32 recorded for the legacy CoopMAC and compared to the value of 0.76 for the DCF protocol. 


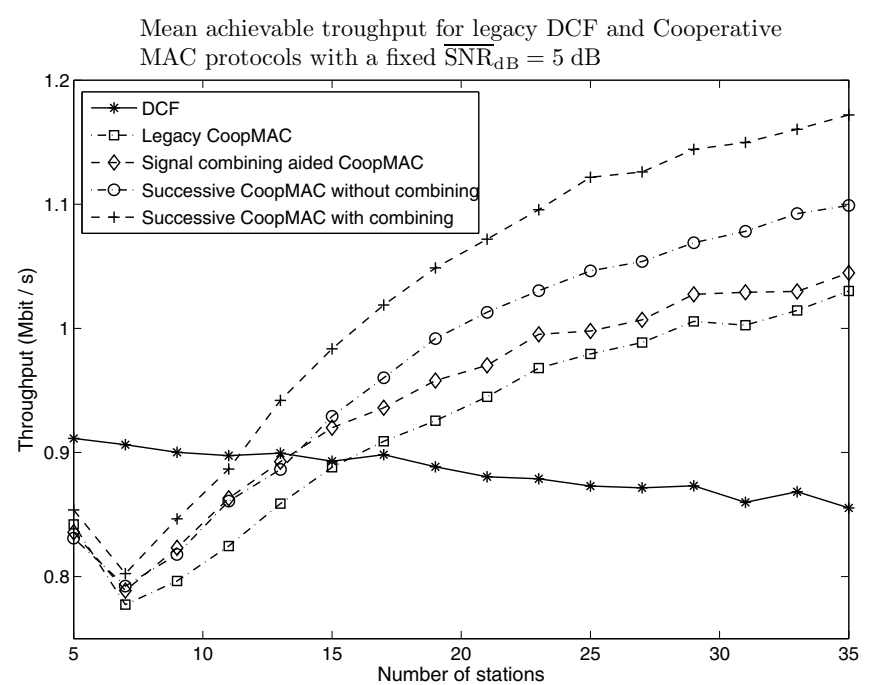

Fig. 3. Mean throughput of the proposed schemes for $\overline{\mathrm{SNR}}=5 \mathrm{~dB}$ at $1 \mathrm{~m}$ over a slowly fading Rayleigh channel.

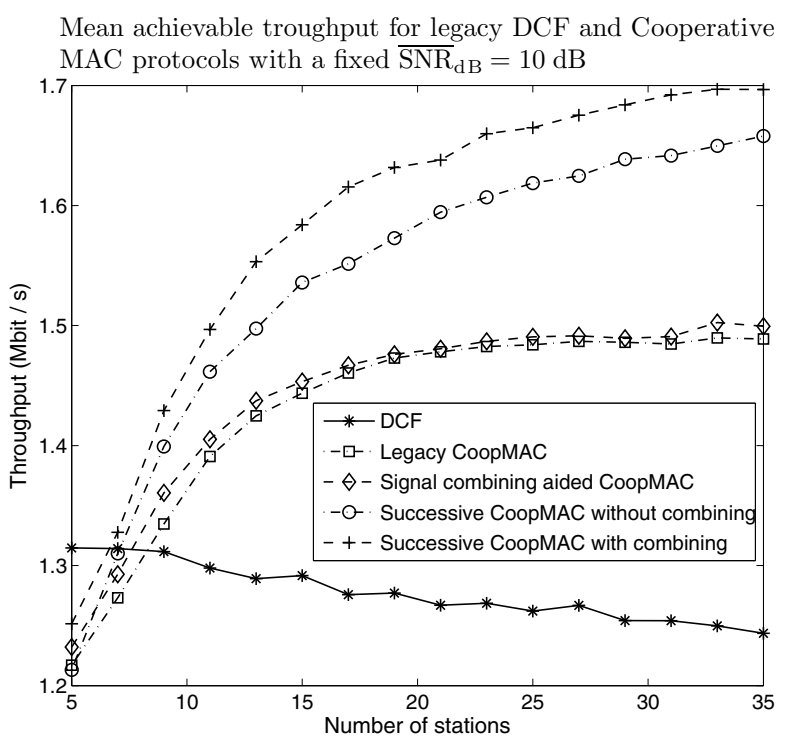

Fig. 4. Mean throughput of the proposed schemes for $\overline{\mathrm{SNR}}=10 \mathrm{~dB}$ at 1 $\mathrm{m}$ over a slowly fading Rayleigh channel.

\section{CONCLUSIONS}

In this study we designed a new cooperative MAC protocol, adopting a cross-layer approach with the introduction of physical layer techniques. A novel transmission rate selection algorithm was amalgamated with efficient LLR combining of the direct and relayed components at the destination. This algorithm allowed us to opt for the best rate, which provided a fixed BLER at the destination, when jointly decoding the frame received from both the source and the relay. We demonstrated that signal combining provides a reduced outage probability and increases the attainable network throughput.

The proposed scheme mitigated the multiplexing loss imposed by the half-duplex constraint of 802.11 stations using a successive relaying protocol relying two relays. We investigated how the two relays should be selected and activated by the protocol. Finally, we
Outage probability for legacy DCF and Cooperative MAC protocols with a fixed $\overline{\mathrm{SNR}}_{\mathrm{dB}}=0 \mathrm{~dB}$

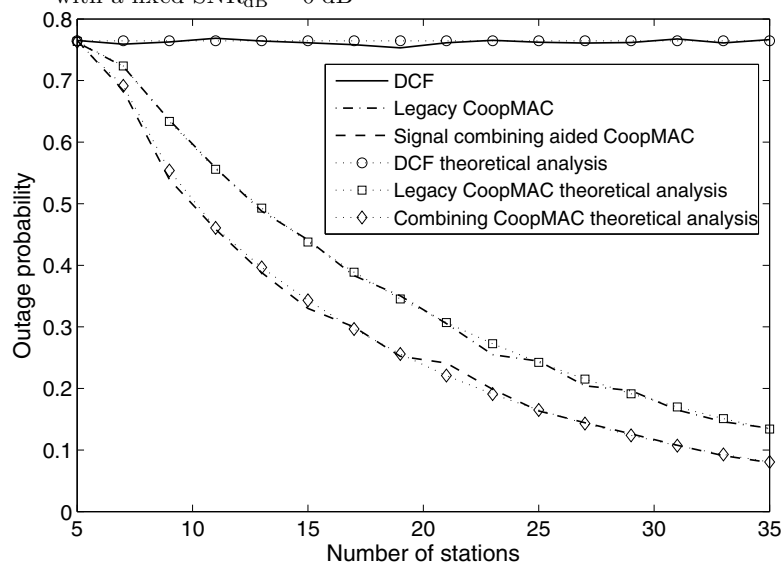

Fig. 5. Outage probability of the proposed schemes for $\overline{\mathrm{SNR}}=0 \mathrm{~dB}$ at 1 $\mathrm{m}$ over a slowly fading Rayleigh channel

showed that invoking two relays has the potential of further increasing the network's throughput gain.

\section{REFERENCES}

[1] L. Hanzo, O. Alamri, M. El-Hajjar, and N. Wu, Near-Capacity MultiFunctional MIMO Systems: Sphere-Packing, Iterative Detection and Cooperation. Wiley-Blackwell, 2009.

[2] H. Shan, W. Zhuang, and Z. Wang, "Distributed cooperative MAC for multihop wireless networks," Communications Magazine, IEEE, vol. 47, pp. 126-133, February 2009.

[3] P. Liu, Z. Tao, Z. Lin, E. Erkip, and S. Panwar, "Cooperative wireless communications: A cross-layer approach," IEEE Wireless Communications, vol. 13, no. 4, pp. 84-92, 2006.

[4] H. Zhu and G. Cao, "rDCF: A relay-enabled medium access control protocol for wireless ad hoc networks," IEEE Transactions on Mobile Computing, vol. 5, no. 9, pp. 1201-1214, 2006.

[5] L. Yi and J. Hong, "A New Cooperative Communication MAC Strategy for Wireless Ad Hoc Networks," Computer and Information Science, ACIS International Conference on, vol. 0, pp. 569-574, 2007.

[6] P. Liu, Z. Tao, S. Narayanan, T. Korakis, and S. Panwar, "CoopMAC: A cooperative MAC for wireless LANs," IEEE Journal on Selected Areas in Communications, vol. 25, no. 2, pp. 340-354, 2007.

[7] Y. Fan, C. Wang, J. Thompson, and H. Poor, "Recovering multiplexing loss through successive relaying using repetition coding," IEEE Transactions on Wireless Communications, vol. 6, no. 12, pp. 4484-4493, 2007.

[8] J. Tee, D. Taylor, and P. Martin, "Multiple serial and parallel concatenated single parity-check codes," IEEE Transactions on Communications, vol. 51, no. 10, pp. 1666-1675, 2003.

[9] F. Babich, "Design of adaptive systems for the fading channel adopting efficient coded modulations," in IEEE International Conference on Communications, 2006. ICC'06, vol. 3, pp. 1433-1438, 2006.

[10] D. M. Rankin and T. A. Gulliver, "Single parity check product codes," IEEE Transactions on Communications, vol. 49, no. 8, pp. 1354-1362, 2001.

[11] F. Babich, "On the performance of efficient coding techniques over fading channels," IEEE Transactions on Wireless Communications, vol. 3, no. 1, pp. 290-299, 2004.

[12] L. Kong, S. X. Ng, R. G. Maunder, and L. Hanzo, "Successive relaying aided near-capacity irregular distributed space-time coding," in IEEE Globecom 2009, November 2009.

[13] L. Kong, S. Ng, and L. Hanzo, "Near-capacity three-stage downlink iteratively decoded generalized layered space-time coding with low complexity," in IEEE Globecom 2008, December 2008. 